\title{
Integral equations of the third kind
}

\author{
by
}

EBERHARD SCHOCK (Kaiserslautern)

\begin{abstract}
Integral equations of the third kind were introduced by D. Hilbert and studied by E. Picard in some very special cases. Here it is shown that in general these equations are ill-posed problems. New and very simple proofs of the convergence properties of the Tikhonov regularization of ill-posed problems and the Landweber iteration are given.
\end{abstract}

1. History. In his fundamental papers on integral equations D. Hilbert [6] introduced the notion of integral equations of the first, second, and of the third kind. A linear integral equation

$$
r(t) x(t)+\int k(t, \tau) x(\tau) d \tau=y(t)
$$

is said to be of the first kind if $r \equiv 0$, of the second kind if $r$ is a non-zero complex constant, and of the third kind if $r$ is a function with zeros in its domain, otherwise the equation is equivalent to an equation of the second kind. Hilbert himself considered the case where $r$ is piecewise constant with values 1 and -1 and with a finite number of jumps and $k$ induces a positive definite operator (polar integral equation). He showed that these equations with some slight modifications have the same properties as the equations of the second kind. In 1910 E. Picard [12] considered the case where the function $r$ is continuous and has a finite number of simple zeros, then with

$$
z(t)=r(t) \cdot x(t)
$$

the equation (1) is equivalent to

$$
z(t)+\int \frac{k(t, \tau)}{r(\tau)} z(\tau) d \tau=y(t) .
$$

He showed that with additional assumptions on the kernel function $k$ (analyticity in some disc) solutions of (2) exist and depend on the value

$$
\gamma=\lim \log \frac{\eta}{\varepsilon}
$$

where the integral in a neighbourhood of a zero $t_{0}$ is split into integrals over $\left(t_{0}-\eta, t_{0}+\varepsilon\right)$ and the complement. Using slightly different methods, 
G. Fubini [2] (1912) considered the same equation as E. Picard. C. Platrier ([14], chapt. V, 27) (1913) considered the case where $r$ has a finite number of zeros of arbitrary order and the kernel is analytic in some disc, and he showed the existence of solutions of (2) again depending on $\gamma$.

Referring these topics, E. Hellinger and O. Toeplitz [5] classified integral equations of the third kind as proper singular equations. In my opinion this classification is not adequate if singular integral equations are equations where the kernel function has singularities. If $r$ is e.g. a characteristic function of a proper subset of positive measure, then (1) is in no sense equivalent to (2). Integral equations of the first and of the third kind are equations of the type

$$
T x=y
$$

where the operator $T$ in general does not have a closed range, therefore they are ill-posed equations.

2. Ill-posedness of integral equations of the third kind. Let $X, Y$ be normed spaces, $T: X \rightarrow Y$ a linear operator. Following J. Hadamard, the problem of solving the equation

$$
\text { ' } T x=y
$$

is said to be well-posed if $T$ meets the conditions

(a) $T$ is surjective,

(b) $T$ is injective,

(c) $T^{-1}$ is continuous.

Otherwise, this problem is ill-posed. If $X$ and $Y$ are complete and if Range $T$ is not closed, then the problem of solving equation (3) is always an ill-posed problem. Then there occur two difficulties: Also if $T$ is injective, $T^{-1}$ is not continuous, and since Range $T$ is of first category in $\overline{\operatorname{Range} T}$, in each neighbourhood of $y \in \operatorname{Range} T$ there are $y_{\delta} \notin \operatorname{Range} T$. Both properties lead to serious computational difficulties. To avoid these problems one replaces the operator $T$ by an operator $T_{\alpha}$ where $\alpha$ is a small numerical parameter and studies the solution $x_{\alpha, \delta}$ of the perturbed equation

$$
T_{\alpha} x_{\alpha, \delta}=y_{\delta}
$$

One method which leads to a well-posed problem is the regularization method of Tikhonov: to compute the minimizer of $\left\|T x-y_{\delta}\right\|$ in some set $W$ with the constraint that $\|x\|$ is minimal. If $X$ and $Y$ are Hilbert spaces, this is equivalent to computing the minimizer $x_{\alpha, \delta}$ of

$$
Q_{\alpha}\left(x, y_{\delta}\right)=\left\|T x-y_{\delta}\right\|^{2}+\alpha\|x\|^{2}, \quad \alpha>0 .
$$

Then

$$
x_{\alpha, \delta}=\left(T^{*} T+\alpha I\right)^{-1} T^{*} y_{\delta}
$$

is the solution of a well-posed problem, since $T^{*} T$ is positive definite.

In general an integral equation of the third kind can be written in the following form: Let $(\Omega, \Sigma, \mu)$ be a finite measure space and $L_{2}=L_{2}(\Omega, \Sigma, \mu)$ the complex Hilbert space. For $r \in L_{\infty}$ the operator of multiplication

$$
M_{r}: L_{2} \rightarrow L_{2} \text { with } M_{r} x=r \cdot x
$$

is a bounded linear operator with $\left\|M_{r}\right\|=\|r\|_{\infty}$. Let $k: \Omega \times \Omega \rightarrow C$ be such that

$$
T_{k}: L_{2} \rightarrow L_{2} \quad \text { with } \quad\left(T_{k} x\right)(t)=\int k(t, \tau) x(\tau) d \tau
$$

is a compact integral operator. Then (1) is equivalent to

$$
\left(M_{r}+T_{k}\right) x=y .
$$

The essential spectrum $\sigma_{e}(T)$ in the sense of M. Schechter [16] is the part of the spectrum $\sigma(T)$ of $T$ which remains invariant under all perturbations of $T$ by compact operators,

$$
\sigma_{e}(T)=\bigcap_{K \text { compact }} \sigma(T+K) .
$$

The essential range $R_{e}(r)$ of an $L_{\infty}$-function $r$ is the set of all $\lambda \in C$ such that for each neighbourhood $V$ of $\lambda$ the inverse $r^{-1}(V)$ of $V$ has positive measure. J. I. Nieto [11] has shown that the essential spectrum $\sigma_{e}\left(M_{r}\right)$ of the operator of multiplication by $r$ is the same as the essential range of $r$. Therefore

$$
\text { If } 0 \in \sigma_{e}\left(M_{r}\right)=R_{e}(r) \text {, then (5) is an ill-posed problem. }
$$

(Actually, Nieto has shown more: one can replace $M_{r}$ by an operator $M_{r_{1}}+r_{2} \cdot S$, where $S$ is a certain operator as a Hilbert transform or a singular integral operator, then a similar result holds. Our following considerations are also valid in this more general case.) If $T$ is a compact integral operator with the polar decomposition

$$
T=\sum \lambda_{j} u_{j} \otimes v_{j},
$$

then E. Picard [13] characterized the range of $T$ as the set of all $x \in L_{2}$ such that

$$
x=\sum \zeta_{j} v_{j} \quad \text { and } \quad \sum \lambda_{j}^{-2}\left|\zeta_{j}\right|^{2}<\infty .
$$

Similarly in the case of integral equations of the third kind, the range of $T$ $=M_{r}+T_{k}$ can be characterized by means of the spectral decomposition of

$$
|T|=\left(T^{*} T\right)^{1 / 2}
$$


and the polar decomposition

$$
T=U \cdot|T|
$$

with a partial isometry $U$. Since the non-uniqueness of the solution of equation (3) is a minor problem (it can be solved by using the techniques of generalized inverses, see C. Groetsch [4]) I will always assume that the operator

$$
T=M_{r}+T_{k}
$$

is injective.

3. Tikhonov regularization. It is well known that the regularized solutions converge to the solution $\hat{x}$ of (3) (see e.g. A. N. Tikhonov, V. Y. Arsenin [19] or V. K. Ivanov, V. V. Vasin, V. P. Tanana [7] for the proof and further references) Here I will give a very simple proof of this fact.

THEOREM 1. Let $T: H_{1} \rightarrow H_{2}$ be an injective linear operator between Hilbert spaces $H_{1}$ and $H_{2}$. Let $y \in \operatorname{Range} T$. Then for $\alpha>0$ the minimizers $x_{\alpha}$ of

$$
Q_{\alpha}(x, y)=\|T x-y\|_{H_{2}}^{2}+\alpha\|x\|_{H_{1}}^{2}
$$

converge to the solution $\hat{x}$ of (1).

Proof. Since $x_{\alpha}$ is given by

$$
x_{\alpha}=\left(T^{*} T+\alpha I\right)^{-1} T^{*} y,
$$

it follows that

$$
\hat{x}-x_{\alpha}=\left(I-\left(T^{*} T+\alpha I\right)^{-1} T^{*} T\right) \hat{x}, \quad \hat{x}-x_{\alpha}=\alpha\left(T^{*} T+\alpha I\right)^{-1} \hat{x} .
$$

For each $\hat{x} \in$ Range $T^{*} T$ for an appropriate $\hat{u} \in H_{1}$

$$
\hat{x}-x_{\alpha}=\alpha\left(T^{*} T+\alpha I\right)^{-1} T^{*} T \hat{u} .
$$

Since

$$
\left\|\alpha\left(T^{*} T+\alpha I\right)^{-1} T^{*} T\right\| \leqslant \alpha,
$$

pointwise convergence for each $\hat{x}$ in the dense set Range $T^{*} T$ follows, therefore by the principle of uniform boundedness for each $y \in \operatorname{Range} T$ one has

$$
\lim _{\alpha \rightarrow 0} x_{\alpha}=\hat{x}
$$

If $\varphi:[0,1] \rightarrow R$ is a monotone increasing function with $\varphi(0)=0$, then the operators

$$
\varphi(\alpha)^{-1} \alpha\left(T^{*} T+\alpha I\right)^{-1}, \quad \alpha>0
$$

are not uniformly bounded, therefore the set of all $\hat{x} \in H_{1}$ with

$$
\left\|\hat{x}-x_{\alpha}\right\|=\left\|\alpha\left(T^{*} T+\alpha I\right)^{-1} \hat{x}\right\|=. O(\varphi(\alpha))
$$

is of first category in $H_{1}$. This shows that in general the convergence is arbitrarily slow. With additional assumptions on the solution $\hat{x}$ which can be understood as smoothness conditions in the case of integral operators, the proof of the above theorem leads to a simple proof of a theorem of V. A. Morozov [10].

Corollary 2. If $\hat{x} \in \operatorname{Range} T^{*} T$, then $\left\|\hat{x}-x_{\alpha}\right\|=O(\alpha)$.

If $\hat{x} \in$ Range $T^{*}$, then $\left\|\hat{x}-x_{\alpha}\right\|=O(\sqrt{\alpha})$.

Proof. The first part is a consequence of (7). To prove the second part one observes that

$$
\hat{x}-x_{\alpha}=\alpha\left(T^{*} T+\alpha I\right)^{-1} T^{*} v=\alpha T^{*}\left(T T^{*}+\alpha I\right)^{-1} v .
$$

With a unitary operator $V$ we have

$$
T^{*}=V\left(T T^{*}\right)^{1 / 2}
$$

therefore

$$
\left\|\hat{x}-x_{\alpha}\right\|=\sqrt{\alpha} \cdot\left\|\left(\alpha T T^{*}\right)^{1 / 2}\left(T T^{*}+\alpha I\right)^{-1} v\right\| .
$$

Since

$$
\left\|\left(\alpha T T^{*}\right)^{1 / 2}\left(T T^{*}+\alpha I\right)^{-1}\right\|=\sup _{\lambda \in \sigma\left(T T^{*}\right)} \frac{\sqrt{\alpha \lambda}}{\lambda+\alpha} \leqslant \frac{1}{2}
$$

also the second part is proven.

This corollary can be generalized in the following way.

THeOREM 3 [17]. If $\hat{x} \in \operatorname{Range}\left(T^{*} T\right)^{p}, 0<p<1$, then

$$
\left\|\hat{x}-x_{\alpha}\right\|=o\left(\alpha^{p}\right) .
$$

Proof. With an appropriate $\hat{u} \in H_{1}$

$$
\hat{x}-x_{\alpha}=\alpha\left(T^{*} T+\alpha I\right)^{-1}\left(T^{*} T\right)^{p} \hat{u} .
$$

Since for $\alpha>0$

$$
\left\|\alpha^{1-p}\left(T^{*} T+\alpha I\right)^{-1}\left(T^{*} T\right)^{p}\right\|=\sup \frac{(\lambda / \alpha)^{p}}{\lambda / \alpha+1}<\infty,
$$

the property

$$
\left\|\hat{x}-x_{\alpha}\right\|=O\left(\alpha^{p}\right)
$$

follows. On the other hand, since

$$
\left\|\left(T^{*} T+\alpha I\right)^{-1} T^{*} T\right\| \leqslant 1,
$$


for each $\hat{v} \in \operatorname{Range}\left(T^{*} T\right)^{1-p}$

$$
\left\|\alpha^{1-p}\left(T^{*} T+\alpha I\right)^{-1} T^{*} T \hat{v}\right\|=O\left(\alpha^{1-p}\right) .
$$

The density of Range $\left(T^{*} T\right)^{1-p}$ in $H_{1}$ implies then

$$
\left\|\hat{x}-x_{\alpha}\right\|=o\left(\alpha^{p}\right)
$$

If Range $T$ is not closed then in each $\delta$-neighbourhood of $y \in \operatorname{Range} T$ there is a $y_{\delta} \notin \operatorname{Range} T$ with

$$
\left\|y-y_{\delta}\right\| \leqslant \delta
$$

So the convergence of the minimizers $x_{\alpha, \delta}$ of

$$
Q_{\alpha}\left(x, y_{\delta}\right)=\left\|T x-y_{\delta}\right\|^{2}+\alpha\|x\|^{2}
$$

depends both on the convergence of $\alpha \rightarrow 0$ and $\delta \rightarrow 0$. V. A. Morozov [10] has given a relation between $\alpha$ and $\delta$ in order to obtain convergence. We will give here a simple proof of this fact.

THEOREM 4. Let T be an injective linear operator between Hilbert spaces. Let $y \in$ Range $T,\left\{y_{\delta} \in H, \delta>0\right\}$ be a set of elements with $\left\|y-y_{\delta}\right\| \leqslant \delta$. If $\alpha>0$ and $x_{\alpha, \delta}$ is the minimizer of $Q_{\alpha}\left(\cdot, y_{\delta}\right)$, then

$$
\lim _{\alpha, \delta \rightarrow 0} \frac{\delta^{2}}{\alpha}=0 \quad \text { implies } \quad \lim _{\alpha, \delta \rightarrow 0}\left\|\hat{x}-x_{\alpha, \delta}\right\|=0 .
$$

Proof. With a unitary operator $V$ one has $T^{*}=V\left(T T^{*}\right)^{1 / 2}$, therefore and

$$
x_{\alpha, \delta}=\left(T^{*} T+\alpha I\right)^{-1} T^{*} y_{\delta}=V\left(T T^{*}\right)^{1 / 2}\left(T T^{*}+\alpha I\right)^{-1} y_{\delta}
$$

$$
\left\|x_{\alpha}-x_{\alpha, \delta}\right\|=\left\|\left(T T^{*}\right)^{1 / 2}\left(T T^{*}+\alpha I\right)^{-1}\left(\dot{y}-y_{\delta}\right)\right\| .
$$

Since $\left\|\left(\alpha T T^{*}\right)^{1 / 2}\left(T T^{*}+\alpha I\right)^{-1}\right\| \leqslant \frac{1}{2}$,

and

$$
\left\|x_{\alpha}-x_{\alpha, \delta}\right\| \leqslant \frac{1}{2} \frac{\delta}{\sqrt{\alpha}}
$$

$$
\begin{aligned}
\lim \left\|\hat{x}-x_{\alpha, \delta}\right\| & \leqslant \lim \left\|\hat{x}-x_{\alpha}\right\|+\lim \left\|x_{\alpha}-x_{\alpha, \delta}\right\| \\
& \leqslant \lim \left\|\hat{x}-x_{\alpha}\right\|+\lim \frac{\delta}{2 \sqrt{\alpha}}=0 .
\end{aligned}
$$

Corollary 5. If $T$ is injective and for the solution $\hat{x}$ of (1) we have $\hat{x} \in \operatorname{Range}\left(T^{*} T\right)^{p}$ for some $0<p \leqslant 1$, then the optimal rate of convergence is given for $\alpha=\gamma \cdot \delta^{2 /(2 p+1)}, \gamma>0$ by

$$
\left\|\hat{x}-x_{\alpha, \delta}\right\|=O\left(\delta^{2 p /(2 p+1)}\right)
$$

Proof. By Theorem 3 and Theorem 4

$$
\left\|\hat{x}-x_{\alpha, \delta}\right\|=o\left(\alpha^{p}\right)+O\left(\frac{\delta}{\sqrt{\alpha}}\right)=O\left(\delta^{2 p /(2 p+1)}\right)
$$

if $\alpha$ is chosen as $\gamma \cdot \delta^{2 /(2 p+1)}$.

This result is due to C. W. Groetsch [3] in the case $p=1$ and to myself [17] in the case $p<1$ for compact operators $T$.

4. Tikhonov regularization for self-adjoint operators. Since self-adjoint operators have real spectrum, for purely imaginary parameters $\tau$ one has

$$
\left\|(T+\tau I)^{-1}\right\| \leqslant 1 /|\tau| \text {. }
$$

This leads to the following convergence result:

THEOREM 6. Let $T$ be an injective self-adjoint operator, $\tau$ purely imaginary. Let $y \in \operatorname{Range} T$; then for the solution $x_{\tau}$ of

we have

$$
T x_{\tau}+\tau x_{\tau}=y
$$

$$
\lim _{\mathfrak{\tau} \rightarrow 0}\left\|\hat{x}-x_{\tau}\right\|=0
$$

Proof.

$$
\hat{x}-x_{\tau}=\left(I-(T+\tau I)^{-1} T\right) \hat{x}=\tau(T+\tau I)^{-1} \hat{x} .
$$

The operators

$$
\tau(T+\tau I)^{-1}, \quad i \tau \in \boldsymbol{R} \backslash\{0\}
$$

are uniformly bounded, and for each $\hat{x} \in$ Range $T$

$$
\left\|\tau(T+\tau I)^{-1} \hat{x}\right\|=\left\|\tau(T+\tau I)^{-1} T \hat{u}\right\|=O(|\tau|),
$$

therefore since Range $T$ is dense in $H$, by the principle of uniform boundedness the convergence is proven.

The following theorem again requires the dependence of the choice of $\delta$ and $\tau$ to obtain convergence.

THEOREM 7. Let The injective self-adjoint; $\hat{x} \in \operatorname{Range}|T|^{p}, 0<p \leqslant 1, y_{\delta} \in H$, $\left\|y-y_{\delta}\right\| \leqslant \delta$ and $x_{\tau, \delta}$ the solution of

$$
T x_{\tau, \delta}+\tau x_{\tau, \delta}=y_{\delta}, \quad i \tau \in \boldsymbol{R} \backslash\{0\} .
$$

Then

$$
\tau=i \gamma \cdot \delta^{1 /(p+1)}, \gamma>0, \quad \text { implies } \quad\left\|\hat{x}-x_{\tau, \delta}\right\|=O\left(\delta^{p /(p+1)}\right)
$$


Proof. If $\hat{x} \in \operatorname{Range}|T|^{p}=\operatorname{Range}\left(T^{2}\right)^{p / 2}$, then

$$
\begin{gathered}
\hat{x}-x_{\tau}=\tau(T+\tau I)^{-1}|T|^{p} \hat{u}, \\
\left\||\tau|^{1-p}(T+\tau I)^{-1}|T|^{p}\right\|=\sup _{\dot{\lambda} \in \sigma(T)} \frac{|\lambda / \tau|^{p}}{|\lambda / \tau+1|}<\infty ;
\end{gathered}
$$

therefore

$$
\left\|\hat{x}-x_{\tau}\right\|=O\left(|\tau|^{p}\right)
$$

From

$$
\left\|x_{\tau}-x_{\tau, \delta}\right\|=\left\|(T+\tau I)^{-1}\left(y-y_{\gamma}\right)\right\| \leqslant \frac{\delta}{|\tau|}
$$

follows

$$
\begin{aligned}
\left\|\hat{x}-x_{\tau, \gamma}\right\| & \leqslant\left\|\hat{x}-x_{\tau}\right\|+\left\|x_{\tau}-x_{\tau, \delta}\right\| \\
& =O\left(|\tau|^{p}\right)+O\left(\frac{\delta}{|\tau|}\right)=O\left(\delta^{p /(p+1)}\right)
\end{aligned}
$$

if $|\tau|=\gamma \cdot \delta^{1 /(p+1)}$

5. Finite-dimensional approximations. The finite-dimensional approximation of the solution $\hat{x}$ of (3) needs a further assumption on the choice of the parameter $\alpha$ to obtain convergence. Let $\left(X_{n}\right)$ be a sequence of subspaces of $H_{1}$ with $\operatorname{dim} X_{n}=n, \cup X_{n}$ dense in $H_{1}, P_{n}: H_{1} \rightarrow X_{n}$ the orthonormal projection. Let $x_{\alpha, n}$ be the minimizer of

in $X_{n}$. Then

$$
Q_{\alpha}(x)=\|T x-y\|^{2}+\alpha\|x\|^{2}
$$

$$
x_{\alpha, n}=\left(P_{n} T^{*} T P_{n}+\alpha P_{n}\right)^{-1} P_{n} T^{*} y .
$$

THEOREM 8. Let $T: H_{1} \rightarrow H_{2}$ be an injective linear operator, $y \in \operatorname{Range} T$. Let $\alpha=\alpha(n)$ be chosen such that

$$
\left\|P_{n} T^{*} T\left(I-P_{n}\right) \hat{x}\right\|=o(\alpha)
$$

where $\hat{x}=T^{-1} y$ is the solution of (3). Then

$$
\lim _{\alpha, n} x_{\alpha, n}=\hat{x} \text {. }
$$

Proof.

$$
\begin{aligned}
x_{\alpha, n}= & \left(P_{n} T^{*} T P_{n}+\alpha P_{n}\right)^{-1} P_{n} T^{*} T \hat{x} \\
= & \left(P_{n} T^{*} T P_{n}+\alpha P_{n}\right)^{-1} P_{n} T^{*} T P_{n} \hat{x}+ \\
& +\left(P_{n} T^{*} T P_{n}+\alpha P_{n}\right)^{-1} P_{n} T^{*} T\left(I-P_{n}\right) \hat{x} .
\end{aligned}
$$

From (8) it follows that the second term $x_{\alpha, n}^{(2)}$ tends to zero. For the first term $x_{\alpha, n}^{(1)}$ we have

$$
P_{n} \hat{x}-x_{\alpha, n}^{(1)}=\alpha\left(P_{n} T^{*} T P_{n}+\alpha P_{n}\right)^{-1} P_{n} \hat{x}
$$

The operators

$$
\alpha\left(P_{n} T^{*} T P_{n}+\alpha P_{n}\right)^{-1} P_{n}, \quad n \in N, \alpha>0,
$$

are uniformly bounded; for all $\hat{x} \in \operatorname{Range} T^{*} T$ we have

$$
\begin{aligned}
& \| \alpha\left(P_{n} T^{*} T P_{n}+\alpha P_{n}\right)^{-1} P_{n} T^{*} T P_{n} \hat{u}+\alpha\left(P_{n} T^{*} T P_{n}+\alpha P_{n}\right)^{-1} \times \\
& \times P_{n} T^{*} T\left(I-P_{n}\right) \hat{u}\|\leqslant \alpha \cdot\| \hat{u}\|+\| T^{*} T\|\cdot\|\left(I-P_{n}\right) \hat{u} \| \rightarrow 0 ;
\end{aligned}
$$

therefore, since Range $T^{*} T$ is dense, by the principle of uniform boundedness for all $\hat{x} \in H_{1}$

$$
\lim \left(\hat{x}-x_{\alpha, n}\right)=\lim \left(\hat{x}-P_{n} \hat{x}\right)+\lim \left(P_{n} \hat{x}-x_{\alpha, n}^{(1)}\right)+\lim x_{\alpha, n}^{(2)}=0 .
$$

If $y_{\delta}$ does not belong to Range $T$, then from

$$
\hat{x}-x_{\alpha, n, \delta}=\hat{x}-x_{\alpha, n}+\left(P_{n} T^{*} T P_{n}+\alpha P_{n}\right)^{-1} P_{n} T\left(y-y_{\delta}\right)
$$

it follows as in the proof of Theorem 4 that

$$
\left\|\hat{x}-x_{\alpha, n, \delta}\right\| \leqslant\left\|\hat{x}-x_{\alpha, n}\right\|+\delta / 2 \sqrt{\alpha}
$$

which implies convergence if the approximation of $y$ by $y_{\delta}$ is chosen such that $\left\|y-y_{\delta}\right\| \leqslant \delta(n)$ and

$$
\lim _{n \rightarrow \infty} \frac{\delta^{2}(n)}{\alpha(n)}=0 .
$$

Corollary 9. Let $T: H_{1} \rightarrow H_{2}$ be an injective linear operator, $\left\{y_{\delta}, \delta>0\right\}$ a set of approximations of $y \in \operatorname{Range} T$ with $\lim y_{\delta}=y$. If $\alpha=\alpha(n)$ is chosen such that

$$
\left\|P_{n} T^{*} T\left(I-P_{n}\right) T^{-1} y\right\|_{.}=o(\alpha)
$$

and $\delta=\delta(n)$ is chosen such that

$$
\delta(n)=o(\sqrt{\alpha(n)}),
$$

then the sequence $x_{\alpha, n, \delta}$ of minimizers of

$$
\left\|T x-y_{\delta}\right\|^{2}+\alpha\|x\|^{2}
$$

in $X_{n}$ converges to the solution $\hat{x}$ of $T x=y$.

6. Landweber iteration. In $1951 \mathrm{~L}$. Landweber [8] proposed an iterative method for solving integral equations of the first kind. Here I will give a very 
simple proof of this result, which can also be used to simplify the proofs of the related results of $\mathrm{O}$. N. Strand [18].

THEOREM 10. Let $T$ be an injective linear operator in a Hilbert space $H$. Then for $y \in \operatorname{Range} T$ the Landweber iteration

$$
x_{n+1}=x_{n}+\beta T^{*}\left(y-T x_{n}\right), \quad x_{0}=0,
$$

converges to the solution $\hat{x}$ of (1) if $\beta$ is chosen with $0<|1-\beta \lambda|<1$ for $\lambda \in \sigma\left(T^{*} T\right) \backslash\{0\}$.

Proof. From (9) follows by induction

$$
x_{n}=\left[I-\left(I-\beta T^{*} T\right)^{n}\right] \hat{x}
$$

since

$$
\begin{aligned}
x_{n+1} & =\left[I-\left(I-\beta T^{*} T\right)^{n}\right] \hat{x}+\beta T^{*} T\left[\hat{x}-\left(I-\left(I-\beta T^{*} T\right)^{n}\right) \hat{x}\right] \\
& =\left[I-\left(I-\beta T^{*} T\right)^{n}\right] \hat{x}+\beta T^{*} T\left(I-\beta T^{*} T\right)^{n} \hat{x} \\
& =\left[I-\left(I-\beta T^{*} T\right)^{n+1}\right] \hat{x}
\end{aligned}
$$

therefore

$$
\hat{x}-x_{n}=\left(I-\beta T^{*} T\right)^{n} \hat{x}
$$

The operators

$$
\left(I-\beta T^{*} T\right)^{n}, \quad n \in N,
$$

are uniformly bounded. For $\varepsilon>0$ let $\sigma_{1}$ be the set of all spectral values $\lambda$ of $T^{*} T$ with $|\lambda| \leqslant \varepsilon$ and $\sigma_{2}=\sigma\left(T^{*} T\right) \backslash \sigma_{1}$. Let $P\left(\sigma_{1}\right)$ and $P\left(\sigma_{2}\right)$ be the corresponding spectral projections. Then for each $\hat{x} \in$ Range $T^{*} T, \hat{x}=T^{*} T \hat{u}$

$$
\left\|\left(I-\beta T^{*} T\right)^{n} T^{*} T P\left(\sigma_{1}\right) \hat{u}\right\| \leqslant \sup _{\lambda \in \sigma_{1}}|1-\beta \lambda|^{n}|\lambda| \cdot\|\hat{u}\| \leqslant \varepsilon\|\hat{u}\|
$$

and

$$
\left\|\left(I-\beta T^{*} T\right)^{n} T^{*} T P\left(\sigma_{2}\right) \hat{u}\right\| \leqslant \sup _{\lambda \in \sigma_{2}}|1-\beta \lambda|^{n}\|\hat{x}\| .
$$

Since $\sup _{\lambda \in \sigma_{2}}|1-\beta \lambda|<1$, there is an $n_{0} \in N$ such that for all $n \geqslant n_{0}$

$$
\sup _{\lambda \in \sigma_{2}}|1-\beta \lambda|^{n}\|\hat{x}\| \leqslant \varepsilon
$$

This implies

$$
\left\|\hat{x}-x_{n}\right\| \leqslant\left\|\left(I-\beta T^{*} T\right)^{n} T^{*} T P\left(\sigma_{1}\right) \hat{u}\right\|+\left\|\left(I-\beta T^{*} T\right)^{n} T^{*} T P\left(\sigma_{2}\right) \hat{u}\right\|(\|\hat{u}\|+1) \cdot \varepsilon .
$$

Since $T^{*} T$ is injective, Range $T^{*} T$ is dense in $H$, and the principle of uniform boundedness implies

$$
\lim x_{n}=\hat{x}
$$

for each $\hat{x} \in H$.
7. Concluding remarks. In the theorems stated in the previous sections the self-adjointness of $T^{*} T$ resp. $T$ plays an important role. They can be generalized to operators in Banach spaces which admit a similar spectral theory as self-adjoint operators. A class of such operators is the class of scalar type operators, see H. R. Dowson [1]. Theorem 6 is also true for $H$-operators in the sense of A.S. Markus [9] with dense range or for operators with a real numerical range.

\section{References}

[1] H. R. Dowson, Spectral Theory of Linear Operators, Academic Press, 1978.

2] G. Fubini, Sulle equazioni integrali di terza specie di Emile Picard, Acc. Naz. Lincei (Roma) Rendiconti 5. Ser. tom 21 (1912), 325-330.

[3] C. W. Groetsch, On the order of accuracy of Tikhonov regularization, J. Optim. Theory Appl. 44 (1984).

[4] -, Generalized Inverses of Linear Operators, Dekker, 1977.

[5] E. Hellinger and O. Toeplitz, Integralgleichungen und Gleichungen mit unendlich vielen Unbekannten, Enzyklopädie der Math. Wissenschaften II C13, Leipzig 1928.

[6] D. Hilbert, Grundzüge einer allgemeinen Theorie der linearen Integralgleichungen, Leipzig 1912.

[7] V. K. Ivanov, V. V. Vasin and V. P. Tanana, Theory of Linear Ill-Posed Problems and Its Applications (in Russian), Nauka, Moscow 1978.

[8] L. Land we ber, An iteration formula for Fredholm integral equations of the first kind, Amer. J. Math. 73 (1953), 615-624.

[9] A. S. M ark us, Some criteria for the completeness of a system of root vectors of a linear operator in a Banach space, Amer. Math. Soc. Transl. (2) 85 (1969), 51-91.

[10] V.A. Morozov, Choice of parameter for the solution of functional equations by the regularization method, Sov. Math. Dokl. 8 (1967), 1000-1003.

[11] J. I. Niet o, On Fredholm operators and the essential spectrum of singular integral operators, Math. Ann. 178 (1968), 62-77.

[12] E. Picard, Un théorème général sur certaines équations intégrales de troisième espèce, C. R. Acad. Sci. Paris 150 (1910), 489-491; 152 (1911), 1545-1547; 153 (1911), 529-531, 615-617.

[13] -, Sur un théorème général relatif aux équations intégrales de première espèce et sur quelques problèmes de physique mathématique, Rend. Circ. Mat. Palermo 29 (1910), 79-97.

[14] C. Platrier, Sur les mineurs de la fonction déterminante de Fredholm et sur les systèmes d'équations intégrales linéaires, J. Math. Pures Appl. 9 (1913), 233-304.

[15] R. Schatten, Norm Ideals of Completely Continuous Operators, Springer, 1960

[16] M. Schechter, On the essential spectrum of an arbitrary operator, J. Math. Anal. Appl. 13 (1966), 205-215.

[17] E. Schock, On the order of accuracy of Tikhonov regularization, J. Optim. Theory Appl. 44 (1984).

[18] O. N. Strand, Theory and methods related to the singular function expansion and Landwebers iteration for integral equations of the first kind, SIAM J. Numer. Anal. 11 (1974), 798-825.

[19] A. N. Tikhonov and V. Y. Arsenin, Solutions of Ill-Posed Problems, Wiley, 1977.

Received May 17, 1983

(1896) 